\title{
Phenomenal identity and form perception in an infant
}

\author{
T. G. R. BOWER \\ HARVARD UNIVERSITY
}

Gestalt psychologists described in some detail the stimulus conditions which determine which parts of an array will be seen as units possessing "phenomenal identity." These same stimulus conditions, often called the Gestalt laws, were held to be effective determinants of form perception too. A previous study indicated that only one of the Gestalt laws, common fate, was an effective determinant of phenomenal identity in human infants of less than 16 weeks of age. The present study using a more powerful technique shows that another of the Gestalt laws, good continuation, is an effective determinant of phenomenal identity and form perception in infants of 36 days of age. A third variable, proximity, is not effective in this age range.

Perhaps the most primitive ability of any perceptual system is the ability to partial an array of stimulation into segregated units whose parts cohere with one another, remaining independent of the parts of other units. For vision, Koffka (1936) posed the problem as, "Why is it that we see things rather than the spaces between them?" This is the problem of phenomenal identity. Gestalt psychologists, such as Koffka, treated it as a psychophysical problem, seeking to discover what properties of a visual array caused some segments to be seen as coherent units separable from the rest of the array. The resulting stimulus variables were entitled Gestalt laws. Perhaps it is the dignity of the title "law" which has sheltered these psychophysical formulations from further investigation, for little has been done with them since the pioneering Gestaltist investigations. In particular little has been done to investigate their origins, whether in phylogeny or ontogeny. Brunswik (1956) suggested that a process of correlation learning within the lifetime of an individual could explain the effectiveness of the Gestalt laws. Bower (1965) attempted to test this theory by testing the effectiveness of three laws with infants of various ages. Only one, common fate, the tendency to see segments which move together in phase as coherent, was effective with the younger infants. The result thus seemed to confirm Brunwik's empiricism against Gestalt psychologists' nativism. However, subsequent experiments have shown some flaws in the original. Bower (1965) found that infants are insensitive to pictorial information. The dynamic flow of information given in motion seems to be essential for them. It is thus possible that the apparent primacy of common fate resulted from no more than the fact that the display exemplifying it was the only one which involved motion. The displays exemplifying the others were presented as still projections on a screen so that not even head movements could produce motion gradients. Accordingly, the experiment was redone using solid models, which yield motion gradients during head movements, to exemplify the laws which were previously ineffective.

Six groups of four infants served as subjects. All of the infants were 36 days of age at testing. An operant conditioning technique was used. The technique used was modelled after a technique invented by Siqueland (1965). The operant response was a suck of high positive pressure. A sealed nipple was inserted in the infant's mouth. The resulting sucks were transduced and amplified by a schwarzer polygraph. The output from the polygraph was fed into a Schwarzer amplitude discriminator, whose output controlled a counter and the display equipment described below. During the experiment the subject sat on his mother's or an experimenter's lap. The subject-holder was responsible for keeping the nipple in the subject's mouth. Fifteen in. from the subject's face at a $45^{\circ}$ angle to his line of sight was placed a half silvered (30/30) mirror. Immediately behind the mirror at right angles to the line of sight was a translucent screen, behind which a motion picture projector was placed. Parallel to the subject's line of sight was a white peg-board which was used as a stimulus holder. The peg-board was illuminated by fluorescent tubes at top and bottom giving it a luminance of $52 \mathrm{ft} .-\mathrm{L}$. Its edges were baffled so that the subject could see the stimulus only as reflected in the half-silvered mirror. The only other illumination in the room came from a dim corridor light. The output from the amplitude discriminator through a stepping switch extinguished the peg-board light and started the movie projector running for a 10-sec. period, at the end of which the projector stopped and the peg-board light came on again. The projector lamp was never off, so that a stopped frame of the movie was continuously visible behind the CS. For rest periods and stimulus changing both sets of lights were extinguished. The movie shown was of an older child at play. The peg-board stimulus thus served as conditioned stimulus (CS), the high pressure suck as conditioned response (CR) and the 10-sec. movie presentations as reinforcement.

Two CS were used. The first was a black wire equilateral triangle, side $35 \mathrm{~cm}$, thickness of wire $5 \mathrm{~mm}$ with a $7 \mathrm{~cm}$ diameter, $50 \mathrm{~cm}$ long, round, black rod 

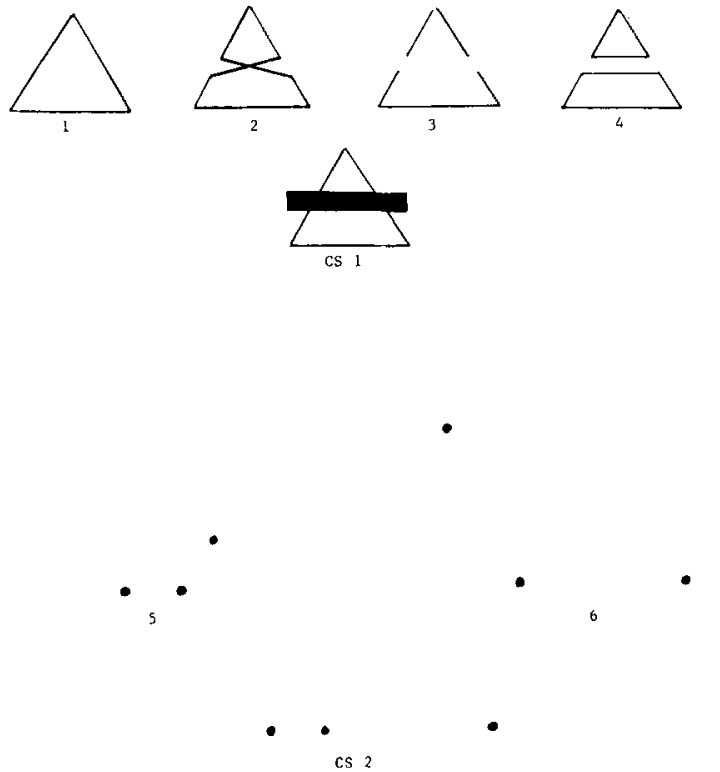

Fig. 1. The conditioning and test stimuli used in these experiments.

placed over it. The second CS consisted of three 2.5 $\mathrm{cm}$ diameter black discs, mounted in a row with an interval of $5 \mathrm{~cm}$ between the left hand pair and $15 \mathrm{~cm}$ between the right hand pair.

Groups $1,2,3$, and 4 were trained with the first CS, and the groups 5 and 6 with the second CS. The training procedure was the same for all groups. Daily 30 45 min. sessions were aimed at. The first session was spent in shaping the response, initially with continuous reinforcement, later with reinforcement on a variable ratio (VR) schedule on which every third response on average was reinforced. On the second day the VR3 schedule was maintained and a discrimination between presence and absence of the CS was formed. The Terrace (1963) fading procedure was used. The lights illuminating the peg-board were switched off for 1-sec. periods between responses. This period was gradually extended to $10 \mathrm{sec}$. During these dark periods a response would not have been reinforced had one occurred. In fact, none occurred. The terminal minute of the second day was a discrimination test period. The peg-board lights were extinguished during this period. None of the infants emitted a single response during the test. This was taken as adequate evidence of discrimination.

The first $15 \mathrm{~min}$. of the third day were spent on a VR10 schedule. Thereafter $2 \mathrm{~min}$, of test trials were given. For $1 \mathrm{~min}$. the CS was present without reinforcement, for the second minute a test stimulus was present, again without reinforcement. Half of the infants in each group viewed the presentations in the stated order, half in the reverse order. The test stimulus for each group is shown in Fig. 1. The reasoning guiding these choices is given below. After the $2 \mathrm{~min}$. of testing, 10 further min. of training were given. Then a further 2 min. of testing, with presentation order reversed for each infant, was given, followed by 3 further min. of training on the VR10 schedule.

These experiments were designed to assess whether two of the Gestalt laws, good continuation and proximity, were effective in the infant subjects. The first CS was designed to assess the effects of good continuation. If infants, like adults, see contours, whose mathematical description in cartesian space is identical, as continuations of one another, then the infants should have seen this CS as a triangle with a bar over it. They should have seen the two visible segments of the triangle as continuations of one another, so that during testing the triangle (in Fig. 1) should have caused the minimal decrement in responding. If on the other hand, good continuation were not effective but if common fate were, if that is to say, the separation between bar and triangle were sufficient for head movements to produce different rates of displacement of their retinal projections, then they should have seen the display as an indeterminate figure with a bar over it; that is, they should have seen the top part of the triangle as connected with the bottom half, but been unable to specify the nature of the connection. If this were so the figures 1 and 2 of Fig. 1 should have been equally ineffective in suppressing responses. If again neither of these variables were effective, then 3 and 4 should have had the least effect, since they at least had no contours which were not present in the $\mathrm{CS}$.

The second CS was designed to assess the effectiveness of the variable, proximity. The relevant Gestalt law may be stated as follows. In a visual field containing three or more identical elements, any pair of elements which are nearer to one another than either is to any other element will be seen as a unit. Thus the CS used here, labelling the dots $a, b, c$, should be seen as ( $a b), c$, with $a$ and $b$ forming a single entity. If this variable were effective one should expect transfer stimulus 5 of Fig. 3 to produce less response decrement than transfer stimulus 6 , since the former does not violate the unity of (ab) whereas transfer stimulus 6 does so.

The results are summarized in Table 1 . As can

Table 1.

\begin{tabular}{ccc} 
Mean Response Rate per Min. to: & CS & Test Stimulus \\
\hline Group & & \\
1 & 51.00 & 34.00 \\
2 & 53.00 & 12.00 \\
3 & 49.00 & 14.00 \\
4 & 52.00 & 12.25 \\
5 & 50.00 & 13.50 \\
6 & 50.25 & 14.00 \\
\hline
\end{tabular}


be seen there, proximity had no effect on responding. The two transfer stimuli elicited equally few responses. There was no significant difference between them. The situation was quite different with good continuation which seemed to be highly effective in that transfer stimulus a, which exemplified good continuation, elicited significantly more responses than any of the other three transfer stimuli ( $p<.001$ by $t$-test), which in turn did not differ significantly from one another. Only on the hypothesis that good continuation is an effective variable can one comprehend this pattern of results.

The conclusions of the previous study must thus be revised. Proximity still seems ineffective but it does appear that good continuation can be an effective variable with young infants. It is worth noting that good continuation functioned here not only as a determinant of phenomenal identity, but also as a determinant of form perception. Not only did the infants "know" that the top of the triangle was connected to the bottom; they also knew the nature of the connection. This indicates a rather high degree of perceptual organization at this comparatively early age, rather more than most current theories of perceptual development would lead one to believe.

\section{References}

Bower, T, G. R. Phenomenal identity in infants. Psychon. Sci. $1965,3,323-324$.

Bower, T. G. R. Stimulus variables determining space perception in infants. Science, $1965,149,88-89$.

Brunswik, E. P'rception and the representative design of psychological experiments. University of California Press, 1956.

Koffka, K. Principles of Gestalt psychology. New York: Routledge 1936.

Siqueland, E. Paper read at 1st conference on infancy. Cambridge 1965.

Terrace, H. Discrimination learning with or without errors. J. exp. Anal. Behav., 1963, 6, 1-27.

(Accepted for publication December 4, 1966.) 